\title{
PISA 2012: Ansiedad y Bajo Rendimiento en Competencia Matemática
}

\section{PISA 2012: Anxiety and Low Performance Mathematics Competence}

\author{
Esperanza Bausela Herreras ${ }^{1}$
}

\begin{abstract}
Resumen
Las matemáticas resultan críticas para el desarrollo del individuo. En este trabajo no experimental, descriptivo, se compara el riesgo de tener bajo rendimiento en matemáticas función del nivel de ansiedad en 25.313 estudiantes españoles, considerando distintas variables predictoras vinculadas con el estudiantes y con el docente, de acuerdo a los resultados de PISA 2012. Las variables estudiadas fueron agrupadas en cuatro modelos: ansiedad, estrategias de aprendizaje, activación cognitiva y atribución del fracaso. En todos ellos se incluyó la variable ansiedad. El riesgo de bajo rendimiento varía en función del nivel de ansiedad en los modelos analizados. En todos los modelos ese riesgo disminuye a medida que se reduce el nivel de ansiedad en función de la escala ordinal propuesta. No hay una relación directa entre rendimiento académico y ansiedad, existiendo variables que median entre ambos. Finalmente, debe atenderse a examinar no solo una relación unidireccional sino, además, una relación bidireccional entre ambas variables.
\end{abstract}

Palabras clave: estudios PISA, rendimiento, competencia, ansiedad, estrategias de aprendizaje, atribución al fracaso y activación cognitiva

\begin{abstract}
Mathematics is critical to the development of the individual. They are different variables that can predict poor performance. In this research, not experimental, descriptive, the risk of underperforming depending on the level of anxiety compared considering different predictor variables linked to the learner and teacher. The variables were grouped into four types model: anxiety, learning strategies, cognitive activation and attribution causal of failure. It has been included in the entire model the anxiety variable included. The risk of low performance varies depending on the level of anxiety in the different models analyzed. It is observed that in three models that risk decreases as the anxiety level is reduced according to the proposal ordinal scale. There is no direct relationship between academic achievement and anxiety, there are variables that mediate between them. Finally, it should be addressed to examine not just a one-way relationship but also a two-way relationship between the two variables.
\end{abstract}

Keywords: PISA studies, performance, competence, anxiety, learning of strategies, attributions to failure and activation cognitive

\footnotetext{
${ }^{1}$ Universidad Pública de Navarra (UPNA). Departamento de Ciencias de la Salud. Área de Psicología Evolutiva y de la Educación. Campus de Arrosadía, Edificio de los Magnolios C.P. 31006 Pamplona (Iruña), España. Tel.: +34948169464 Correo: esperanza.bausela@unavarra.es
} 


\section{Introducción}

Un gran número de escolares experimentan dificultades en las matemáticas (Shalev, Auerbach, Manor, \& Cross, 2000). Se desconoce si estas dificultades obedecen, por ejemplo, a un retraso o a un déficit en los procesos neurocognitivos.

Actualmente se pone énfasis en diversas variables que tratan de explicar el por qué de estas dificultades, por ejemplo, en el estudio de procesos cognitivos de orden superior - funciones ejecutivas - que se han asociado con procesos implicados en el desempeño en matemáticas (Bull \& Scerif, 2001; Presentación, Siegenthaler, Pinto, Mercader, \& Miranda, 2015). Así, por ejemplo, la memoria de trabajo ha sido ampliamente estudiada en niños con dificultades específicas de aprendizaje de las matemáticas (Cragg, Keeble, Richardson, Roome, \& Gilmore, 2017; Geary, Hoard, \& Hamson, 1999; Presentación et al., 2015).

Otra variable de índole cognitivocomportamental es la ansiedad hacia las matemáticas que es un sentimiento de desagrado asociado con la manipulación numérica y con la resolución de problemas matemáticos (Richardson \& Suinn, 1972). Ahmed, Minnaert, Kuyper y van der Werf (2012) operacionalizan este constructo en tres dimensiones: atención en clase, estudiar o hacer tareas escolares en casa y rendimiento en el test de Pekrun (2006).

La competencia matemática pueden generar inquietud, estrés e incluso sensación de impotencia en los jóvenes de 15 años, estando demostrada la relación entre ansiedad y rendimiento (ver Hunt \& Sandhu, 2017). La OECD (2015) en su informe sobre esta materia indica que: (i) esta asociación (negativa) entre el rendimiento y la ansiedad se observa al comparar diferentes alumnos de un mismo sistema educativo; (ii) las mujeres manifiestan mayor ansiedad frente a las matemáticas que los varones; (iii) los estudiantes suelen mostrar más ansiedad cuando la mayoría de sus compañeros de clase obtienen mejores resultados que ellos, existiendo una relación con el centro al que asisten y el grado de desenvoltura que tienen en esa materia frente a sus compañeros. El éxito lo valoran en función de su capacidad para superar a sus iguales.
La ansiedad hacia las matemáticas, la negatividad implícita y el bajo autoconcepto hacia la misma, son algunas de las razones por las que las mujeres pueden estar a la zaga y detrás de sus compañeros varones en matemáticas (ver Nosek \& Smyth, 2011).

La ansiedad ha sido examinada y estudiada en relación con otras variables, por ejemplo, con el autoconocimiento (ver, Ahmed, Minnaert, Kuyper, \& van der Werf, 2012). Morony, Kleitman, Ping, \& Stankov (2013) trataron de predecir el rendimiento considerando el nivel de confianza, autoeficacia, ansiedad y autoconcepto. En los estudios de PISA (2003) se obtuvieron correlaciones significativas entre el nivel de autoconfianza y las puntuaciones en matemáticas. Los tres constructos vinculados con el autoconocimiento son importantes predictores del rendimiento en PISA 2003 (OECD, 2005) y tienen una correlación significativa.

Lee (2009) analiza la ansiedad junto con otros dos constructos - autoconcepto, autoeficacia - que: (i) son independientes, pero están próximos los unos a los otros, (ii) demuestra que hay aspectos particulares de los mismos que son específicos de los países, mostrando patrones de relación específicos en los cuarenta y un países que fueron estudiados y analizados. (iii) Las correlaciones entre estos tres constructos son moderadas, sin embargo, cada uno de ellos parece tener una importante contribución para explicar el rendimiento en matemáticas entre los países y dentro de los mismos países.

En relación con las competencias cognitivas, motivaciones y actitudes, aparecen las estrategias de aprendizaje que ponen en práctica los estudiantes para desarrollar la competencia matemática. Las estrategias son procedimientos que se aplican de modo controlado, dentro de un plan diseñado deliberadamente con el fin de conseguir una meta fijada (Gargallo, 2000). Aeepactamannil (2014) en su estudio con adolescentes hindús concluye que existen asociaciones diferentes con el rendimiento en función de las estrategias empleadas por los estudiantes. Así, los estudiantes que usan frecuentemente estrategias de aprendizaje metacognitivas tienen un rendimiento significativamente mejor en PISA en lectura, matemáticas y ciencias que sus compañeros que 
los que las utilizan con menos frecuencia. En este mismo estudio se compararon los tipos de estrategias utilizados por los estudiantes y su rendimiento, destacando que: (i) los adolescentes que usan frecuentemente estrategias de memorización puntúan significativamente más bajo en PISA (Matemáticas y Ciencias) en comparación aún con los compañeros que usan estrategias metacognitivas con menos frecuencia, (ii) las estrategias de elaboración, sin embargo, se asociaron significativamente y positivamente sólo con el nivel de conocimiento matemático.

Los estudios son concluyentes en relación a la asociación entre el uso de estrategias de aprendizaje (cognitivas y metacognitivas) y el rendimiento académico (e.g. Areepattannil \& Caleon, 2013). En algunos trabajos, el uso de estrategias cognitivas, tales como las estrategias de memorización, se ha relacionado con resultados positivos (e.g. Zimmenrman \& Martinez-Pons, 1986), sin embargo, en otros estudios se ha demostrado una relación negativa (e.g. Areepattamannil \& Caleon, 2013), siendo por consiguiente ambigua la relación.

Es complicado analizar relaciones y extraer conclusiones de estudios que analizan estas variables (mencionadas en páginas precedentes) de forma unidireccional, así, Kwederee (2014) analiza la interconexión entre la ansiedad con indicadores sociodemográficos, autoeficacia y autoconcepto. Los resultados obtenidos indican que los varones tienen un autoconcepto positivo más alto hacia las matemáticas que las mujeres. Almaguer (1998) estima, por su parte, que el éxito o fracaso se atribuye a factores internos que se asocian de forma diferente con el rendimiento académico (Woolfolk, 1995). Por ejemplo, es de conocimiento entre los especialistas que los estudiantes con más altas calificaciones poseen un locus de control interno (Almaguer, 1998).

\section{El presente estudio}

En este contexto, nos planteamos como analizar y comparar el riesgo de tener bajo rendimiento en la competencia matemática en función del nivel de ansiedad, considerando diferentes variables predictoras vinculadas al estudiante: estrategias de aprendizaje y atribución causal al fracaso; y al docente (estrategias docentes de activación cognitiva).
Como hipótesis de investigación nos planteamos que los escolares tienen la misma probabilidad y/o riesgo de tener bajo rendimiento en la competencia matemática evaluada en PISA 2012 en los diferentes modelos propuestos en función de diferentes variables predictoras, estando en todos ellos (modelos analizados) presente la ansiedad.

\section{Método}

Estudio no experimental o ex post facto, de diseño descriptivo.

\section{Participantes}

En el caso de España (objeto del presente estudio) la muestra está constituida por 25.313 jóvenes de 15 años y 902 centros educativos. La técnica de muestreo utilizada es bietápica por conglomerados. Primero se seleccionan los centros educativos (mínimo de 150 por país) y luego los estudiantes (35 alumnos por centro educativo). La muestra total configurada por 373.691 estudiantes. La base de datos está disponible en la Website of the Ministry of Education, Culture and Sport (2012, URL http://www.mecd.gob.es/inee/Bases-de-datos.html Date accessed: 28th August 2016).

\section{Variables}

Las variables han sido agrupadas en torno a tres modelos: (i) Modelo I: ansiedad y estrategias de aprendizaje, (ii) Modelos II: ansiedad, estrategias de aprendizaje y estrategias docentes de activación cognitiva; (iii) Modelo III: ansiedad, estrategias docentes de activación cognitiva y atribución causal del fracaso; (iv) Modelo IV: ansiedad y atribución causal al fracaso (ver Tabla $1)$.

Las variables en función del papel que desempeñan en la investigación se clasifican en:

(i) Variable exógena o independiente: ansiedad, estrategias de aprendizaje, estrategias docentes de activación cognitiva y atribución causal al fracaso.

(ii) Variables endógenas o dependientes: Puntuaciones en la competencia matemática.

La definición de competencia matemática de PISA 2012 incluye tres aspectos interrelacionados: (i) procesos matemáticos que 
Tabla 1. Distribución de variables endógenas y exógenas en los diferentes modelos (elaboración propia)
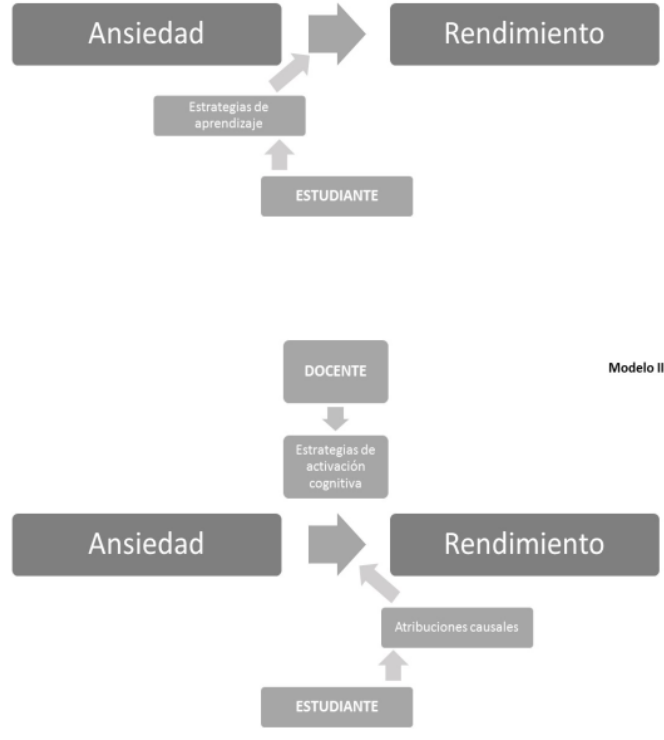

describen lo que hacen los individuos para relacionar el contexto del problema con las matemáticas y de ese modo resolverlo, y las capacidades que subyacen a esos procesos; (ii) contenido matemático específico que va a utilizarse en las preguntas de la evaluación; y (iii) contextos en los que se insertan las preguntas de la evaluación.

Entre los contenidos se han evaluado: (i) cantidad, (ii) espacio, (iii) cambio y relaciones e (iv) incertidumbre y datos. Junto con estos contenidos se han considerado tres procesos: (i) Formulación matemática de las situaciones. (ii) Empleo de conceptos, datos, procedimientos y razonamientos matemáticos. (iii) Interpretación, aplicación y valoración de los resultados matemáticos.

El rendimiento en las tres competencias PISA 2012 es categorizado en diferentes niveles de competencia, siendo el Nivel 1 (nivel inferior) y el Nivel 6 (nivel superior). Así, por ejemplo, se dice que un estudiante tiene un Nivel 1 en competencia Lectora cuando es capaz de localizar información explícita que ocupa un lugar destacado en un texto y que tiene una estructura sintáctica sencilla y un estudiante tiene un Nivel 6 cuando es capaz de realizar inferencias, comparaciones o contrates.
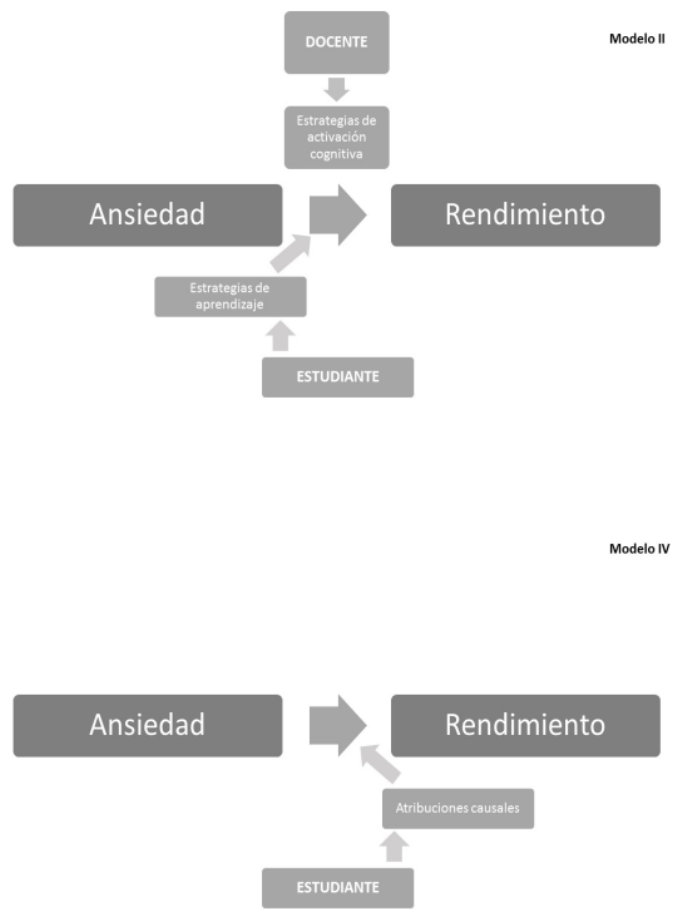

En la competencia matemática el Nivel 1 se identifica como bajo rendimiento (BR) y el resto de niveles como no bajo rendimiento (NBR) (Niveles 2-6). Hemos optado por el Nivel 1 e inferior, ya que son los estudiantes que se encuentran por debajo del percentil 10 (el 90\% de los participantes se sitúan por encima y un $10 \%$ por debajo).

El nivel de competencia matemática ha sido codificado en dos niveles: (i) "SI": bajo rendimiento y (ii) "NO" bajo rendimiento.

\section{Instrumento de recogida de datos}

En PISA 2012, se evalúan tres competencias básicas de Educación Secundaria al finalizar esta etapa (competencia Lectora, Matemática y Científica) en el último curso de Educación Secundaria Obligatoria (Cuarto curso).

Además, incluye un conjunto de información muy extensa sobre el contexto del aprendizaje del alumnado a través de cuestionarios cumplimentados por los propios estudiantes, padres, profesores, directores de los centros educativos que permite conocer otros aspectos.

Así, en este estudio nos hemos centrado en las cuestiones relacionadas con el propio estudiante (ansiedad, estrategias de aprendizaje y atribución 
causal al fracaso) y por parte del docente (estrategias de activación cognitiva).

(I) Estudiante: ansiedad, estrategias de aprendizaje y atribución causal al fracaso.

\section{Ansiedad}

La ansiedad hacia las matemáticas se ha asociado con el bajo rendimiento. En PISA 2012 se ha estudiado con cinco ítems: (i) A menudo me preocupo pensando en tener dificultades en las clases de matemáticas. (ii) Me pongo muy tenso cuando tengo que hacer deberes de matemáticas. (iii) Me pongo muy nervioso al hacer problemas de matemáticas. (iv) Me siento incapaz cuando hago un problema de matemáticas. (v) $\mathrm{Me}$ preocupo cuando pienso que sacaré malas notas en matemáticas.

En su evaluación se ha utilizado una escala tipo Likert: Fuertemente de acuerdo, De acuerdo, En desacuerdo, Fuertemente en desacuerdo y No sabe o no contesta, no válido y perdido.

\section{Estrategias de aprendizaje}

El informe español PISA 2012 está centrado en analizar las cuestiones enfocadas a conocer la frecuencia con la que los estudiantes emplean distintas estrategias en su estudio cotidiano.

Estas estrategias se clasifican en: (i) Estrategias de control: El alumno controla el proceso de aprendizaje y determina en cada momento lo que necesita saber y aquello que desconoce. (ii) Estrategias de reflexión-relación: El estudiante reflexiona sobre lo que estudia y busca relacionar esos conocimientos con otras asignaturas o con la vida real. (iii) Estrategias memorísticas: El estudiante basa su aprendizaje, sobre todo, en memorizar los conceptos y repetir los ejercicios propuestos en clase. Es un cuestionario constituido por cuatro preguntas con tres opciones de respuesta, la primera respuesta corresponde con estrategias de control, la segunda con estrategias de reflexión-relación y la última de ellas con estrategias memorísticas.

\section{Atribución causal al fracaso}

En PISA 2012 se incluyen un cuestionario constituido por seis preguntas con cuatro opciones de respuesta tipo Likert (Muy de acuerdo, De acuerdo, En desacuerdo, Totalmente en desacuerdo). Las preguntas del cuestionario sobre atribuciones al fracaso en matemáticas son: No soy bueno con los problemas de matemáticas, El profesor no explica bien, Malos cálculos, conjeturas..., La materia (matemáticas) es demasiado dura, El/la profesor/a no consigue interesar a los estudiantes y Mala suerte;

(II) Docente: Estrategias de activación cognitiva.

Las estrategias docentes se pueden clasificar, de acuerdo con el estudio PISA 2012 (ver MEC y OCDE, $2013 \mathrm{a}, \mathrm{b}$ ), en estrategias tradicionales o dirigidas por el profesor, estrategias centradas en el estudiante, estrategias de activación cognitiva y, finalmente, estrategias de evaluación formativa.

En este estudio nos centramos en las estrategias de activación cognitiva que son aquellas en las que es el estudiante es quien primero ha de buscar una respuesta a las preguntas que se plantean en clase. En estas estrategias el profesor se convierte en ayudante y motivador, en instigador de la curiosidad del estudiante, fomentando su capacidad de pensar, de buscar formas alternativas de resolver el mismo problema $\mathrm{y}$, en definitiva, estimulando su capacidad crítica. Estas estrategias cognitiva representan la enseñanza basada en paradigmas constructivistas.

La escala de activación cognitiva fue construida usando respuestas que dan los estudiantes a la frecuencia de una serie de situaciones que ocurren con la enseñanza de las matemáticas por parte del docente. La escala de frecuencia es de tipo Likert (siempre o casi siempre, a menudo, a veces, nunca o raramente) y se deriva de la escala propuesta por Baumert et al. (2009).

En estas estrategias el profesor se convierte en ayudante y motivador, en instigador de la curiosidad del estudiante, fomentando su capacidad de pensar, de buscar formas alternativas de resolver el mismo problema y, en definitiva, estimulando su capacidad crítica: Estas estrategias son valoradas con una escala tipo Likert: Siempre o casi siempre, frecuentemente, algunas veces, nunca o rara vez.

\section{Procedimiento}

La aplicación PISA 2012 es realizada por personas especializadas ajenas a los centros educativos en abril y mayo de 2012, en todo el territorio nacional. Los estudios PISA son 
trianuales, esto permite desarrollar estudios longitudinales, analizando la evolución de los distintos sistemas educativos participantes. Se evalúa la competencia Lectora, Matemática y Científica. En la edición correspondiente a 2012, dos terceras partes se centran en la competencia matemática.

\section{Análisis de datos}

Se calcularon los estadísticos descriptivos de las variables independientes y de la variable dependiente principal.

Con el fin de predecir la ocurrencia de bajo rendimiento en función de una serie de variables predictoras vinculadas con factores diversos (ansiedad, estrategias de aprendizaje, atribuciones causales al fracaso y activación cognitiva) optamos por un análisis de regresión logística binaria (Logist Probability Unit).

En los cuatro modelos se ha incluido la variable ansiedad, con el fin de poder comparar, posteriormente, la probabilidad y riesgo de los participantes con diferentes niveles de ansiedad de tener bajo rendimiento en función de las diferentes variables predictoras.

La asociación entre la variable dependiente, rendimiento en la competencia matemática y la variable independiente principal ansiedad, fue calculada con diferentes estadísticos.

\section{Resultados}

Los valores de los estadísticos descriptivos de las variables independiente y dependiente se ofrecen en la Tabla 2.

Los resultados sobre las asociaciones entre variables muestran que (ver Tabla 3):

I. La asociación más fuerte se establece entre "Me siento incapaz cuando hago un problema de matemáticas" y el rendimiento en la competencia matemática $[\chi 2(3, \mathrm{~N}=16577)=671.068, p<.001]$.

II. Los coeficientes obtenidos se podrían calificar de muy bajos, siendo la asociación, muy débil entre la competencia matemática y la ansiedad.

III. Los valores de significación asociados nos indican que hay una relación entre la ansiedad y el nivel de competencia matemática. Esta asociación es muy baja (rango inferior a 0.5 ).
Como se indicara precedentemente, se optó como técnica de análisis de datos por un análisis de regresión logística binaria. Los resultados obtenidos nos indican que no todas las variables tienen el mismo peso en la predicción del bajo rendimiento en la competencia matemática. En la Tabla 4 se presentan los Coeficientes $\beta$ (junto con la probabilidad asociada) y datos resumidos de los cuatro modelos, para la variable bajo rendimiento en la competencia matemática de PISA 2012.

Finalizamos, con el análisis del riesgo que tiene un escolar "prototipo" de tener bajo rendimiento en la competencia matemática en los cuatro modelos propuestos. El riesgo varía en función del nivel de ansiedad en los diferentes modelos analizados.

En todos los modelos - se ha optado por considerar 0 a todas las variables exógenas, salvo la variable relacionada con la ansiedad, con el fin de poder comparar (la probabilidad en función de los diferentes nivel de ansiedad) con las variables de referencia que hemos considerado prototípicas.

Sustituyendo los valores en las variables predictoras que conforman la ecuación, predecimos la probabilidad de tener bajo rendimiento de un "escolar prototípico" en la competencia matemática de PISA 2012 en los cuatro modelos. Como estos valores no son fáciles de interpretar - cuyos valores se encuentran en el rango [0-1] - han sido transformados en porcentajes.

Se observa que en todos modelos ese riesgo disminuye a medida que desciende el nivel de ansiedad en función de la escala ordinal propuesta (fuertemente de acuerdo, de acuerdo, en desacuerdo, fuertemente en desacuerdo), (ver Tabla 5 y Figura 1), existiendo una relación inversa entre el rendimiento en la competencia matemática y el nivel de ansiedad manifestado por el estudiante.

\section{Discusión}

La hipótesis de investigación que guía este estudio sostiene la ausencia de diferencias en la probabilidad y/o riesgo de tener bajo rendimiento en la competencia matemática evaluada en PISA 2012, en diferentes modelos configurados por distintas variables predictoras, estando presente en todos ellos la variable ansiedad.

Los resultados confirman que la ansiedad es una variable que tiene un mayor peso predictivo del bajo rendimiento de la competencia 
Tabla 2. Frecuencia variables explicativas (dependientes) y la variable a explicar (independiente)

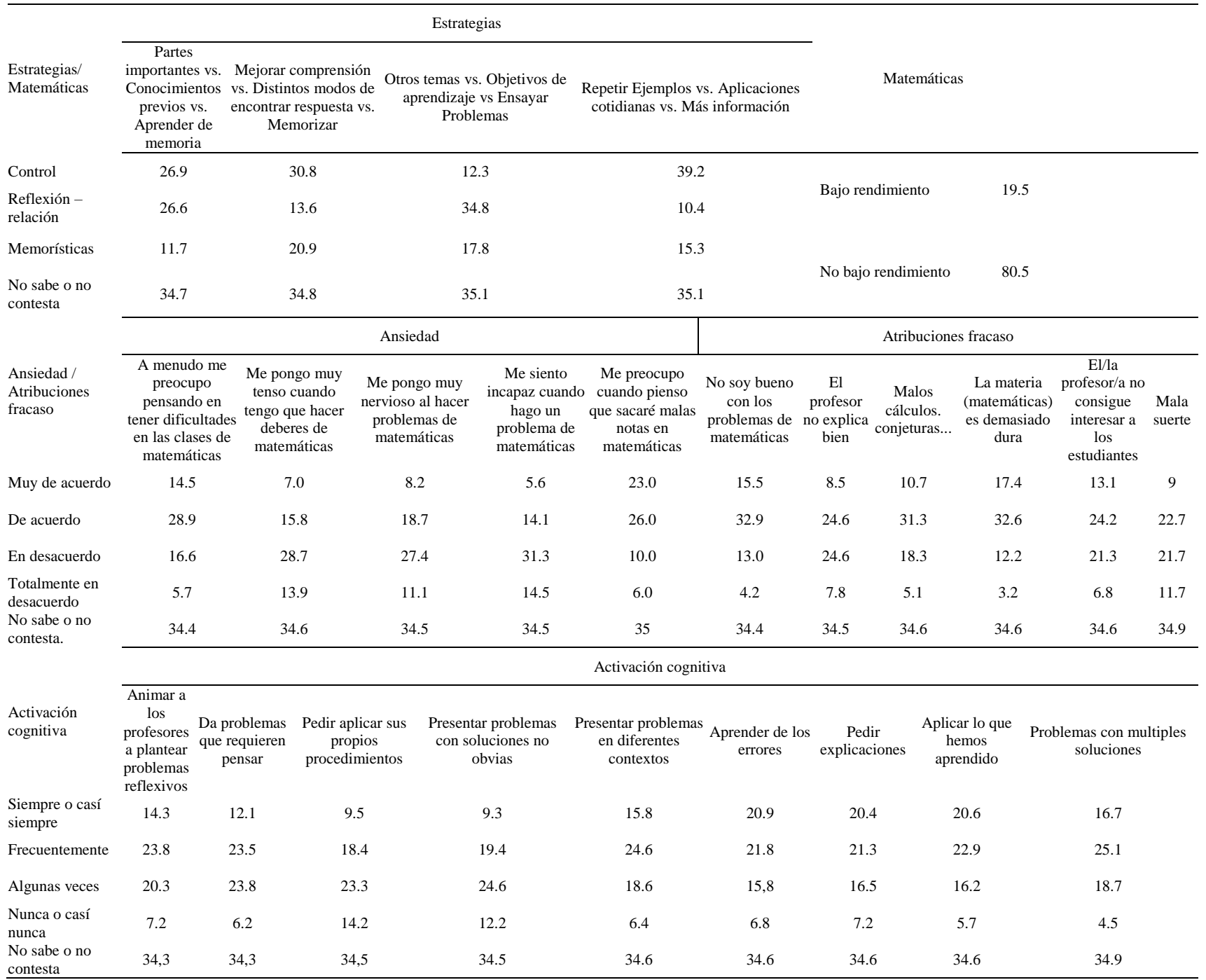

Tabla 3. Pruebas de Chi-Cuadrado y medidas simétricas

\begin{tabular}{lccccc}
\hline Matemáticas & $\begin{array}{c}\text { Preocupación } \\
\text { dificultades }\end{array}$ & Tensión & Nerviosismo & Incapacidad & $\begin{array}{c}\text { Preocupación } \\
\text { malas notas }\end{array}$ \\
\hline Phi & $0.129 * * *$ & $0.188^{* * *}$ & $0.143^{* * *}$ & $0.201 * * *$ & $0.025^{* *}$ \\
V de Cramer & $0.129 * * *$ & $0.188^{* * *}$ & $0.143^{* * *}$ & $0.201 * * *$ & $0.025^{* * *}$ \\
$\begin{array}{l}\text { Coeficiente de } \\
\text { contingencia }\end{array}$ & $0.128^{* * *}$ & $0.185^{* * *}$ & $0.141^{* * * *}$ & $0.197 * * *$ & $0.025^{* *}$ \\
$\begin{array}{l}\text { Chi-cuadrado de } \\
\text { Pearson }\end{array}$ & $276.443(\mathrm{a})^{* * *}$ & $587.859(\mathrm{a}) * * *$ & $338.382(\mathrm{a}) * * *$ & $671.068(\mathrm{a}) * * *$ & $10.482(\mathrm{a})^{* *}$ \\
\hline
\end{tabular}

Nota 1: A menudo me preocupo pensando tener dificultades en las clases de matemáticas; 2: Me pongo muy tenso cuando tengo que hacer deberes de matemáticas; 3: Me pongo muy nervioso al hacer problemas de matemáticas; 4: Me siento incapaz cuando hago un problema de matemáticas; 5 : Me preocupo cuando pienso que sacaré malas notas en matemáticas

Nota 2: (a) 0 casillas (.0\%) tienen una frecuencia esperada inferior a 5. La frecuencia mínima esperada es 2064,03.

$(* * *)$ Diferencias significativas $p<.001(* *)$ Diferencias significativas $p<.01$. (*) Diferencias significativas $p<.05$. (n.s.) No significativa. Fuente: Elaboración propia a partir de PISA 2012 (muestra española).

matemática en dos modelos - Modelo I (estrategias de aprendizaje) y II (estrategias de aprendizaje y activación cognitiva).

En los Modelos III (activación cognitiva y atribución al fracaso) y IV (atribución al fracaso) son las variables relacionadas con la atribución causal las que tienen un mayor poder predictivo.
Estos datos nos permiten afirmar que la ansiedad es una variable que predice de forma diferente el bajo rendimiento en función de la presencia de otras variables predictoras: estrategias de aprendizaje, activación cognitiva y atribución causal del fracaso del escolar. 
Tabla 4. Coeficientes $\beta$ y datos resumidos de los cuatro modelos para la variable bajo rendimiento en la competencia matemática de PISA 2012

\begin{tabular}{|c|c|c|c|c|}
\hline Variables & Modelo I & Modelo II & Modelo III & Modelo IV \\
\hline \multicolumn{5}{|l|}{ Ansiedad } \\
\hline $\begin{array}{l}\text { A menudo me preocupo pensando tener dificultades en las clases } \\
\text { de matemáticas. }\end{array}$ & -0.083 n.s. & -0.083 n.s. & $-0.108^{*}$ & $-0.127 * *$ \\
\hline $\begin{array}{l}\text { Me pongo muy tenso cuando tengo que hacer deberes de } \\
\text { matemáticas }\end{array}$ & $-0.296 * * *$ & $-0.301 * * *$ & $-0.413 * * *$ & $-0.406 * * *$ \\
\hline Me pongo muy nervioso al hacer problemas de matemáticas & $-0.080 *$ & -0.079 n.s. & -0.025 n.s. & $-0.029 *$ \\
\hline Me siento incapaz cuando hago un problema de matemáticas & $-0.342 * * *$ & $-0.349 * * *$ & $-0.408 * * *$ & $-0.39 * * *$ \\
\hline $\begin{array}{l}\text { Me preocupo cuando pienso que sacaré malas notas en } \\
\text { matemáticas }\end{array}$ & $0.226 * * *$ & $0.224 * * *$ & $0.317 * * *$ & $0.349 * * *$ \\
\hline \multicolumn{5}{|l|}{ Estrategias de aprendizaje } \\
\hline $\begin{array}{l}\text { Partes importantes vs. Conocimientos previos vs. Aprender de } \\
\text { memoria }\end{array}$ & $0.098 * *$ & $0.092 *$ & - & - \\
\hline $\begin{array}{l}\text { Mejorar comprensión vs. Distintos modos de encontrar respuesta } \\
\text { vs. Memorizar }\end{array}$ & $0.194 * * *$ & $0.192 * * *$ & - & - \\
\hline Otros temas vs. Objetivos de aprendizaje vs Ensayar Problemas & $-0.098 *$ & -0.086 n.s. & - & - \\
\hline $\begin{array}{l}\text { Repetir Ejemplos vs. Aplicaciones cotidianas vs. Más } \\
\text { información }\end{array}$ & $0.097 *$ & $0.094 * *$ & - & - \\
\hline \multicolumn{5}{|l|}{ Activación cognitiva } \\
\hline Animar a los profesores a plantear problemas reflexivos & - & $-0.165^{* * *}$ & $-0-075^{*}$ & - \\
\hline Dar problemas que requieren pensar & - & -0.078 n.s. & $-0-098 *$ & - \\
\hline Pedir aplicar sus propios procedimientos & - & $-0.197 * * *$ & $-0-041$ n.s. & - \\
\hline Presentar problemas con soluciones no obvias & - & 0.05 n.s. & 0.028 n.s. & - \\
\hline Presentar problemas en diferentes contextos & - & $0.147 * * *$ & $0.106 * *$ & - \\
\hline Aprender de los errores & - & $-0.175^{* * *}$ & $-0.114 * *$ & - \\
\hline Pedir explicaciones & - & -0.067 n.s. & $-0.114 * *$ & - \\
\hline Aplicar lo que hemos aprendido & - & $0.191 * * *$ & $0.256 * * *$ & - \\
\hline Problemas con múltiples soluciones & - & $0.094 *$ & 0.073 n.s. & - \\
\hline \multicolumn{5}{|l|}{ Atribución causal al fracaso } \\
\hline No soy bueno en los problemas de matemáticas & - & - & $-0.087 *$ & $-0.093^{*}$ \\
\hline Los docentes no explican bien & - & - & $0.172 * * *$ & $0.188 * * *$ \\
\hline Malas suposiciones & - & - & 0.02 n.s. & 0.017 n.s. \\
\hline Materia demasiado dura & - & - & $-0.016 \mathrm{n} . \mathrm{s}$. & -0.02 n.s. \\
\hline El profesor no consigue interesar a los estudiantes & - & - & $0.143 * * *$ & $0.159 * * *$ \\
\hline Mala suerte & - & - & $-0.144 * * *$ & $-0.159 * * *$ \\
\hline Constante & -0.039 n.s & 0.146 n.s. & 0.09 n.s & -0.054 n.s \\
\hline Datos resúmenes del modelo & - & - & - & - \\
\hline$-2 \log$ de la verosimilitud & $7106.492^{\mathrm{a}}$ & $6612.391^{\mathrm{a}}$ & $6604.975^{\mathrm{a}}$ & $6995.838^{\mathrm{a}}$ \\
\hline $\mathrm{R}$ cuadrado de Cox y Snell & 0.052 & 0.066 & 0.074 & 0.066 \\
\hline R cuadrado de Nagelkerke & 0.085 & 0.109 & 0.122 & 0.109 \\
\hline$\%$ Clasificado & $81.8 \%$ & $82.4 \%$ & $82.5 \%$ & $82.0 \%$ \\
\hline
\end{tabular}

Nota 1: (a) La estimación ha terminado en el número de iteración 5 porque las estimaciones de parámetro han cambiado en menos de .001 .

Nota 2: Nota: Modelo I: ansiedad y estrategias de aprendizaje. Modelo II: ansiedad, estrategias de aprendizaje y activación cognitiva. Modelo III: ansiedad, activación cognitiva y atribución causal al fracaso. Modelo IV: Ansiedad y atribución causal al fracaso.

(***) Diferencias significativas $p<.001$ (**) Diferencias significativas $p<.01$. (*)Diferencias significativas $p<.05$. (n.s.) No significativa.

Fuente: Elaboración propia a partir de PISA 2012 (muestra española).

Tabla 5. Riesgo de tener bajo rendimiento en matemáticas en PISA 2012 en relación a la ansiedad en los cuatro modelos propuestos

\begin{tabular}{lcccc}
\hline \multirow{2}{*}{ Modelos } & \multicolumn{4}{c}{ Ansiedad } \\
\cline { 2 - 5 } & Fuertemente de acuerdo & De acuerdo & En desacuerdo & $\begin{array}{c}\text { Fuertemente } \\
\text { en desacuerdo }\end{array}$ \\
\hline Modelo I & 28.62 & 13.85 & 6.05 & 2.52 \\
Modelo II & 39.13 & 29.23 & 20.98 & 14.58 \\
Modelo III & 35.05 & 22.55 & 13.58 & 7.81 \\
Modelo IV & 34.14 & 21.18 & 12.23 & 6.74 \\
\hline
\end{tabular}

Nota: Modelo I: ansiedad y estrategias de aprendizaje. Modelo II: ansiedad, estrategias de aprendizaje y activación cognitiva. Modelo III: ansiedad, activación cognitiva y atribución causal al fracaso. Modelo IV: Ansiedad y atribución causal al fracaso.

Fuente: Elaboración propia a partir de PISA 2012 (muestra española). 


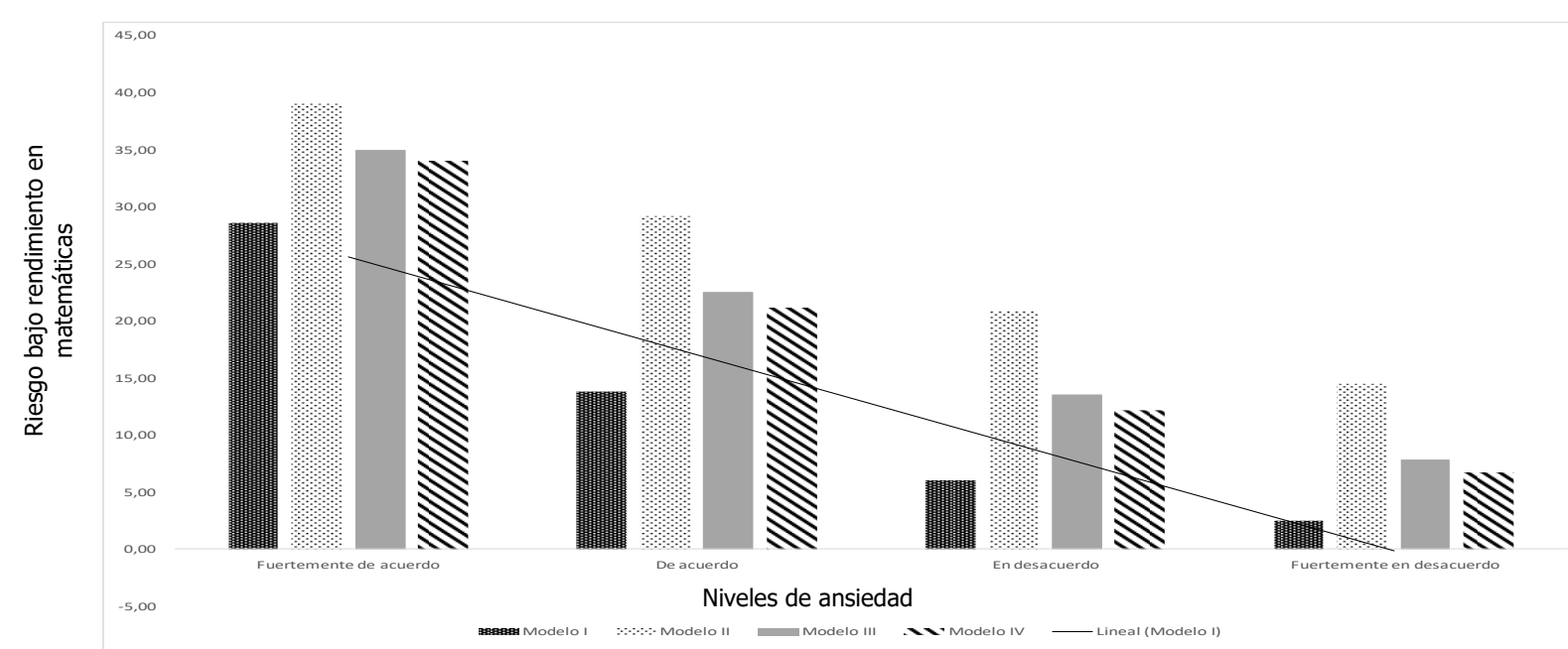

Figura 1. Riesgo de tener bajo rendimiento en matemáticas en PISA 2012 en relación a la ansiedad en los cuatro modelos propuestos y tendencia

Nota: Modelo I: ansiedad y estrategias de aprendizaje. Modelo II: ansiedad, estrategias de aprendizaje y activación cognitiva. Modelo III: ansiedad, activación cognitiva y atribución causal al fracaso. Modelo IV: Ansiedad y atribución causal al fracaso.

Fuente: Elaboración propia a partir de PISA 2012 (muestra española).

Con el estudio diseñado y los análisis efectuados no se puede afirmar que exista una relación lineal y unidireccional entre ansiedad y rendimiento académico, tal y como se estima en ciertos estudios (Ashcraft, \& Kirk, 2001; Ashcraft, \& Moore, 2009; Ashcraft, \& Ridley, 2005; Eysenck, \& Calvo, 1992; Suárez, Núñez, \& Colomé, 2016).

En el estudio realizado sólo podemos afirmar, además, que la variable ansiedad, no predice por si misma el riesgo de tener bajo rendimiento, siendo un conjunto de variables las que permiten predecirlo. Estos resultados se encuentran en consonancia con los resultados obtenidos por otros investigadores que abordan la predicción del rendimiento (Areepattamannil et al., 2016; Ashcraft, \& Kirk, 2001; Chin, Williams, Taylor, \& Harvey, 2017).

En el presente estudio, la ansiedad se ha estudiado en combinación con las estrategias de aprendizaje que utiliza el propio estudiante, teniendo mayor capacidad predictiva que otras variables analizadas y vinculadas con el propio estudiante, por ejemplo, la atribución causal que realiza el estudiante de su rendimiento. Estos resultados nos orientan sobre la necesidad de desarrollar programas que incidan en el manejo de la ansiedad hacia las matemáticas en combinación con el desarrollo de estrategias de aprendizaje (fomentando, por ejemplo, un aprendizaje significativo), incidiendo en el aspecto o esfera cognitiva (ver ejemplo, Ho, Senturk, Lam,
Zimmer, Hong, \& Okamoto, 2000) y no tanto en la atribución causal del rendimiento en matemáticas (Guzman, 2008).

Los resultados obtenidos, además, nos indican que existen diferencias en la predicción del bajo rendimiento en función de los niveles de ansiedad, siendo la relación inversa entre el rendimiento en la competencia matemática y el nivel de ansiedad. Podemos inferir a partir de estos resultados que un nivel de ansiedad es necesario para ser eficientes, pero superado dicho nivel puede entorpecer e incluso interferir de forma negativa el rendimiento (ver Piemontesi \& Heredia, 2009).

En este estudio entre la variable ansiedad y el rendimiento académico en matemáticas, hemos incluido diversas variables cognitivas $y$ comportamentales que median en las mismas. Estos resultados se unen a los obtenidos por otros estudios que señalan el papel que tienen otras variables que median entre la ansiedad y el bajo rendimiento, por ejemplo, el uso de estrategias de aprendizaje (Maloney, Warchter, Risko, \& Fugelsang, 2012; Ramirez, Chang, Maloney, Levine, \& Beilock, 2016), atribución causal (Guzman, 2008), funciones ejecutivas (ver Cragg et al., 2017) o estrategias psicoinstruccionales que aplican los docentes (Jackson \& Leffingwell, 1999). A título de ejemplo, destacamos un reciente metaanálisis (ver Cragg et al., 2017) que destaca una fuerte asociación entre el ejecutivo central de la memoria de trabajo y el rendimiento en matemáticas, o entre la memoria verbal y el 
rendimiento, más que con la memoria visual del clásico modelo de Baddeley y Hitch (1974).

Finalmente, no se puede obviar que la relación entre rendimiento y ansiedad no es unidireccional, como ya hemos comentado en páginas precedentes (ver Karasela, Aydab, \& Tezer, 2010; Max, 1999; Ma \& Xu, 2003) (no era el objetivo de análisis de este estudio). Es necesario desarrollar estudios que exploren una relación bidireccional para poder dar respuesta a las siguientes interrogantes imayor nivel de ansiedad predispone a un bajo rendimiento? o por el contrario ¿es el bajo rendimiento el que predispone a un mayor nivel de ansiedad? A estas preguntas no se puede dar respuesta con el diseño desarrollado en el presente texto, considerando que las variables - ansiedad y rendimiento en matemáticas - están asociadas de forma bidireccional, no siendo posible establecer relaciones de causalidad al desconocer quién es el antecedente, para ello habrá que desarrollar, por ejemplo, un análisis de ecuaciones estructurales.

Es importante entender las variables del presente estudio de forma conjunta y conocer su influencia e incidencia para diseñar propuestas instruccionales que incidan en todas ellas (ver Moenikia \& Zahed-Babelan, 2010; Mora, 2003) y en concreto en aquéllas que hemos detectado que incrementan el riesgo. Las estrategias de activación cognitiva, a título de ejemplo, son sólo una de las estrategias docentes que recomienda la OECD (2013c) para el desarrollo de la competencia matemática. Su efectividad, por otro lado, está vinculada a numerosas variables, como, por ejemplo, la estructuración de los contenidos, el clima del aula o actitudes que manifiesta el estudiante hacia las matemáticas, que deben ser consideradas en futuros estudios.

Éste estudio, en definitiva, analiza la predisposición del riesgo a tener bajo rendimiento en la competencia matemática a partir de la ansiedad en combinación con otras variables. La detección del riesgo de forma precoz es fundamental y puede ayudar a orientar una intervención psicopedagógica que minimice su impacto, apostando claramente por una intervención primaria (proactiva y no reactiva) (ver por ejemplo, Santos Cela, 2008).

Es posible predecir el riesgo de tener dificultades específicas en el aprendizaje de las matemáticas desde la etapa de Educación Infantil (ver Mazzocco \& Thompson, 2005) y al mismo tiempo, recomendable muy necesario identificar el riesgo ya que un rendimiento bajo en matemáticas puede tener un impacto negativo en la adolescencia y en la edad adulta (ver Bausela, prensa).

\section{Limitaciones y perspectivas de futuro}

En este estudio no se han considerado variables intrínsecas al propio sujeto (actitud, expectativas, autoeficacia hacia las matemáticas...) que pueden explicar, por ejemplo, el uso de las estrategias por parte del propio estudiante. Es por ello, que en futuros trabajos podemos desarrollar modelos de ecuaciones estructurales que incluyan dichas variables, que permitan conocer y profundizar no sólo del riesgo sino, además, en la asociación bidireccional de las mismas. Así, por ejemplo, el estudio de Kriegbauma, Jansen y Spinath (2015) confirman que la motivación juega un papel muy importante en la predicción del rendimiento académico. También se ha demostrado que la percepción positiva y las actitudes positivas se asocian con el rendimiento académico (e.g. Chion \& Fraser, 2009). La actitud y la motivación de los estudiantes, son por consiguientes, factores importante a considerar en futuros estudios.

Finalmente, en un mundo globalizado, es importante analizar y comparar los resultados obtenidos con la muestra española con el resto de países participantes en los estudios PISA. Zhang, Khan y Tahirsylaj (2015) sugieren que la cultura es un importante factor de predicción cuando se comparan y contrastan sistemas escolares diferentes.

\section{Aspectos éticos}

Se ha garantizado el anonimato y la confidencialidad de los datos.

\section{Agradecimientos}

Este estudio fue finalizado durante la estancia de investigación desarrollada en julio de 2016 en el Instituto Nacional de Evaluación Educativa dependiente del Ministerio de Educación, Cultura y Deporte (Madrid, España). 


\section{Referencias}

Ahmed, W., Minnaert, A., Kuyper, H., \& van der Werf, G. (2012). Reciprocal relationships between math self - concept and math anxiety. Learning and Individual Differences, 22, 385-389. doi:10.1016/j.lindif.2011.12.004.

Almaguer, T. (1998). El desarrollo del alumno: Características y estilos de aprendizaje. México: Trillas.

Areepattamannil, S., \& Caleon, I. S. (2013). Relationships of cognitive and metacognitive learning strategies to mathematics achievement in four high-performing East Asian education systems. The Journal of Genetic Psychology, 174, 696-702. doi: 10.1080/00221325.2013.799057

Areepattamannil, S. et al. (2016). International note: Prediction of mathematics work ethic and performance from behavioral, normative, and control beliefs among Qatari adolescents. Journal of Adolescence 46, 38-44. doi: 10.1016/j.adolescence.2015.10.016

Areepattamannil, S., \& Lee, D.H.L. (2014). Linking immigrant parents' educational expectations and aspirations to their children's school performance. The Journal of Genetic $\begin{array}{llll}\text { Psychology, } \quad \text { 175, } & 51-\end{array}$ doi: 10.1080/00221325.2013.799061

Areepattamannil, S., Melkonian, M., \& Swe, M. (2015). International note: Exploring differences in native and immigrant adolescent's mathematics achievement and dispositions towards mathematics in Qatar. Journal of Adolescence, 40, 11-13. doi: 10.1016/j.adolescence.2014.12.010.

Ashcraft, M. H., \& Kirk, E. P. (2001). The relationships among working memory, math anxiety, and performance. Journal of Experimental Psychology: General, 130, 224237. doi: 10.1037/0096-3445.130.2.224.

Ashcraft, M. H., \& Moore, A.M. (2009) Math anxiety and affective drop in performance. Journal of Psychoeducational Assessment, 27(3), 197-205. doi: 10.1177/0734282908330 580

Ashcraft, M. H., \& Ridley S. K. (2005). Math anxiety and its cognitive consequences. A tutorial review. En J.I.D. Campbell,
Handbook of mathematical cognition (pp. 315-327). New York: Psychological Press.

Baddeley, A. D., \& Hitch, G. J. (1974). Working memory. In G. A. Bower (Ed.). Recent advances in learning and motivation (Vol. 8). New York: Academic Press.

Bandura, A. (1997). The anatomy of stages of change. American Journal of Health Promotion, 12, 8-10. doi: 10.4278/0890-117112.1.8

Bausela, E. (prensa). Risk low math performance PISA 2012: Impact of assistance to Early Childhood Education and other possible cognitive variables. Acta de Investigación Psicológica.

Brunello, G., \& Rocco, L. (2013). The effect of immigration on the school performance of natives: Cross country evidence using PISA test scores. Economics of Education Review, 32, 234-246. doi:10.1016/j.econedurev.2012.1 0.006

Bull, R., \& Scherif, G. (2001). Executive functioning as a predictor of children's mathematics ability: Inhibition, switching, and working memory. Developmental Neuropsychology, 19, 273- 293. doi: 10.1207/S15326942DN1903_3

Chin, E. C. H., Williams, M., Taylor, J., \& Harvey, S.T. (2017). The influence of negative affect on test anxiety and academic performance: An examination of the tripartite model of emotions. Learning and Individual Differences 54, 1-8. doi: 10.1016/j.lindif.2017 .01 .002

Chionh, Y. H., \& Fraser, B. J. (2009). Class room environment, achievement, attitudes and selfesteem in geography and mathematics in Singapore. International Research in Geographical and Environmental Education, 18, 29-44. doi: 10.1080/10382040802591530

Cragg, L., Keeble, S., Richardson, S., Roome, H. E., \& Gilmore, C. (2017). Direct and indirect influences of executive functions on mathematics Achievement. Cognition, 162, 12-26. doi: 10.1016/j.cognition.2017.01.014

Eysenck, M. W., \& Calvo, M. G. (1992). Anxiety and performance: The processing efficiency theory. Cognition and Emotion, 6, 409-434. doi: 10.1080/02699939208409696. 
Gargallo, B. (2000). Procedimientos. Estrategias de aprendizaje. Su naturaleza, enseñanza y evaluación. Valencia: Humanidades Pedagogía.

Gutiérrez Calvo, M., \& Avero, P. (1995). Ansiedad, estrategias auxiliares y comprensión lectora: Déficit de procesamiento versus falta de confianza. Psicothema, 7(3), 569-578. Recuperado de http://www.psicothema.com/pdf/1003.pdf.

Guzmán, D. (2008). La relación entre la ansiedad y la atribución causal en la población universitaria. Revista Eos, 2, 1-13.

Ho, H., Senturk, D., Lam, A. G., Zimmer, J.M., Hong, S., \& Okamoto, Y. (2000). The affective and cognitive dimensions of math anxiety: A cross-national study. Journal for Research in Mathematics Education, 31 (3), 362-379. doi: 10.2307/749811

Hunt, T. E., \& Sandhu, K. K. (2017). Endogenous and exogenous time pressure: Interactions with mathematics anxiety in explaining arithmetic performance. International Journal of Educational Research 82, 91-98. doi: 10.1016/j.ijer.2017.01.005

INEE (2014a). Perseverando hacia el éxito en los estudios: PISA 2012 y las CC.AA. Educainee. Especial Autonomías. Pisa in Focus, 3.

INEE (2014b). Motivación para aprender matemáticas y PISA 2012: El caso de las CC.AA. españolas. Educainee. Especial Autonomías. Pisa in Focus, 4.

Jackson, C. D., \& Leffingwell, R. J. (1999). The role of instructors in creating math anxiety in students from kindergarten through college. The Mathematics Teacher, 92(7), 583-586. doi: no disponible.

Karasela, N., Aydab, O., \& Tezer, M. (2010). The Relationship between mathematics anxiety and mathematical problem solving skills among primary school students. Procedia Social and Behavioral Sciences, 2, 58045807. doi: 10.1016/j.sbspro.2010.03.946

Kazelskis, R. (1998). Some dimensions of mathematics anxiety: A factor analysis across instruments. Educational and Psychological Measurement, 58, 623-633. doi: $10.1177 / 0013164498058004006$

Kriegbaum, K., Jansen, M., \& Spinath, B. (2015). Motivation: A predictor of PISA's mathematical competence beyond intelligence and prior test achievement. Learning and Individual Differences, 43, 140-148. doi: 10.1016/j.lindif.2015.08.026

Kvedere, L. (2014). Mathematics self-efficacy, self-concept and anxiety among $9^{\text {th }}$ grade students in Latvia. Social and Behavioral Sciences, 116, 2687-2690. doi: 10.1016/j.sbspro.2014.01.636

Lee, J. (2009). Universals and specifics of math self-concept, math self-efficacy, and math anxiety across 41 PISA 2003 participating countries. Learning and Individual Differences, 19, 355-365. doi: 10.1016/j.lindi f.2008.10.009

Ma, X. (1999). A Meta analysis of the relationship between anxiety toward mathematic and achievement in mathematic. Journal for Research in Mathematic Education, 30 (5), 520-540. doi: 10.2307/749772

Ma, X., \& Xu. J. (2003). The causal ordering of mathematics anxiety and mathematic achievement a longitudinal panel analysis. Journal of Adolescence, 27(2), 165-179. doi: 10.1016/j.adolescence.2003.11.003

Maloney, E. A., Warchter, S. Risko, E. F., \& Fugelsang, J. A. (2012). Reducing the sex difference in math anxiety: The role of spatial processing ability. Learning and Individual Differences 22, 380-384. doi:10.1016/j.lindif. 2012.01.001

Mazzocco, M. M., \& Thompson, R. E. (2005). Kindergarten predictors of math learning disability. Learning Disability Research and Practice, 20(3), 142-155. doi:10.1111/j.15405826.2005.00129.x.

Miller, H., \& Bichsel, J. (2004). Anxiety, working memory, gender, and math performance. Personality and Individual Differences, 37(3), 591-606. doi:10.1016/j.paid.2003.09.029

Moenikia, M., \& Zahed-Babelan, A. (2010). A study of simple and multiple relations between mathematics attitude, academic motivation and intelligence quotient with mathematics achievement. Procedia Social and Behavioral Sciences, 2, 1537-1542. doi: 10.1016/j.sbspro.2010.03.231

Mora, C. D. (2003). Estrategias para el aprendizaje y la enseñanza de las matemáticas. Revista de Pedagogía, 24 (70), 
181-272. Recuperado en 12 de febrero de 2017, de http://www.scielo.org.ve/scielo.php? script=sci_arttext\&pid=S0798-

$97922003000200002 \& \operatorname{lng}=\mathrm{es} \& \operatorname{tlng}=\mathrm{es}$.

Moronoy, S., Kelitman, S., Ping, Y., \& Stankov, L. (2013). Predicting achievement: Confidence vs self-efficacy, anxiety, and selfconcept in Confucian and European countries. International Journal of Educational Research 58, 79-96. doi:10.1016/j.ijer.2012.1 1.002

Nosek, B. A., \& Smyth, F. L. (2011). Implicit social cognitions predict sex differences in math engagement and achievement. American Educational Research Journal, 48, 11251156. doi: $10.3102 / 0002831211410683$

OECD (2005). PISA 2003. Technical Report. Organization for economic co-operation and development

OECD (2014a). ¿Tienen los estudiantes la motivación para lograr el éxito? Pisa in Focus, 37(3).

OECD (2014b) ¿Está relacionada la agrupación y selección de estudiantes en distintos centros educativos con su motivación para aprender? Pisa in Focus, 39 (5).

OECD (2015). ¿Te ponen nervioso las matemáticas? Pisa in Focus, 48(2).

Pekrun, R. (2006). The control value theory of achievement emotions: Assumptions, corollaries, and implications for educational research and practice. Educational Psychology Review, 18, 315-341. doi:10.1007/s10648-006-9029-9.

Piemontesi, S. E., Heredia, E. D., Furlan, L. A., Sánchez-Rosas, J., \& Martínez, M. (2012). Ansiedad ante los exámenes y estilos de afrontamiento ante el estrés académico en estudiantes universitarios. Anales de Psicología, 28(1), 89-96. Recuperado de http://revistas.um.es/analesps/article/view/140 562.

Presentación, M.J., Siegenthaler, R., Pinto, V., Mercader, J. y Miranda, A. (2015). Competencias matemáticas y funcionamiento ejecutivo en preescolar: evaluación clínica y ecológica. Revista de Psicodidáctica, 20(1), 65-82. doi: 10.1387/RevPsicodidact.11086

Ramirez, G., Chang, H., Maloney, E.A., Levine, S.C., \& Beilock, S.L. (2016). On the relationship between math anxiety and math achievement in early elementary school: The role of problem solving strategies. Journal of Experimental Child Psychology 141, 83-100. doi: 10.1016/j.jecp.2015.07.014

Richardson, F.C., \& Suinn, R.M. (1972).The mathematics anxiety rating scale: psychometric data. Journal of Counseling Psychology, 19, 551-554. doi: 10.1037/h0033 456

Santos Cela, J. L. (2008). Aproximación a los problemas de atención en la edad escolar a partir de la evaluación neuropsicológica y su relación con el trastorno de aprendizaje del cálculo. Orientación de programas de intervención educativa. Tesis doctoral inédita. León: Universidad de León.

Shalev, R. S. (2004). Developmental dyscalculia. Journal of Child Neurology, 19, 765-771. doi: 10.1177/08830738040190100601

Suárez, M., Núñez, M. I., \& Colomé, A. (2016). Math anxiety: A review of its cognitive consequences, psychophysiological correlates, and brain bases. Cognitive, Affective, \& Behavioral Neuroscience, 16 (1), 3-22. doi: 10.3758/s13415-015-0370-7.

Suzanne, M., Kleitman, S., Ping Lee, Y., \& Stankov, L. (2013). Predicting achievement: Confidence vs self - efficacy, anxiety, and self-concept in Confucian and European countries. International Journal of Educational Research, 58, 79-96. doi: 10.1016/j.jjer.2012.11.002

Weinstein, C. E., \& Mayer, R. E. (1986). The teaching of learning strategies. En M. C.Wittrock (Ed.), Handbook of research on teaching. Nueva York: McMillan.

Woolfolk, A. (1995). Psicología Educativa. México: Prentice Hall Hispanoamericana, S. A..

Zhang, L. Khan, G., \& Tahirsylaj, A. (2015). Student performance, school differentiation and world cultures: Evidence from PISA 2009. International Journal of Educational Development 42, 43-53. doi: 10.1016/j.ijedudev.2015.02.013

Zimmerman, B.J., \& Martinez-Pons, M. (1986). Development of a structured interview for assessing students use of self-regulated learning strategies. American Educational Research Journal, 23, 614-628. 